\title{
Over Enhancement Inhibition in Sky Region Based on Dark Channel
}

\author{
Fang Ding*, Cailiang Du and Xiaojing Song \\ College of electronic information and automation, Civil Aviation University of China, Tianjin, China \\ ${ }^{*}$ Corresponding author
}

\begin{abstract}
The dark channel de-fog algorithm has a landmark significance in the field of single image fogging removal. In the original algorithm, the sky region is prone to over enhancement. Existing improved algorithms has to take lots of time and space. In order to improve the effect of defogging and make sure the operation efficiency, this paper presents a sky region overgrowth suppression algorithm, which is based on the phenomenon of that these three channel values in sky area are approaching the maximum. First, the RGB channel in the original image is separated, then histogram equalization is got. Second, a proposed limiting algorithm is used to calculate the oversaturation grayscale information in the sky region, and then the transmission rate is obtained based on the correction of the dark channel de-fog algorithm. Finally, the output image is generated. The validity of the algorithm in this paper is verified. The suppression algorithm and the original algorithm is evaluated by using the visible side evaluation indicators. It is shows that the ratio of supersaturated pixels has been significantly reduced with slightly affecting new visible edge ratio and balanced gradient ratio. The over enhancement suppression algorithm can effectively suppress sky over enhancement. It is proved that the suppression algorithm can effectively correct the transmittance of the sky region in fog images, and be suitable for occasions where require high speed.
\end{abstract}

Keyword—dark-channel; sky area over enhanced;single image defogging; limiting

\section{INTRODUCTION}

In foggy weather, tiny particles such as water droplets and solid particles are suspended in the air.The scattering of atmospheric light through these tiny particles can blur the image obtained by various sensors, and impact the shooting effect and theresolving power in various monitoring systems. The removal of fog in the image has an important research significance.

At present, the single image defog algorithm can be broadly two categories: 1, algorithm does not take into account the cause of image degradation, you get sensitive information in the image after processing the data, but it's easy to distort, such as histogram equalization method [1], Retinex [2] etc. The other algorithm consider the degradation of the image, according to the physical model to elicit the original image, the resulting image is more realistic [3-5], but it is difficult to estimate the atmospheric light and light transmittance.

The main methods in the field of single image de-fog are following. According to the statistical law proposed of the phenomenon of image contrast with no fog is better than fog one, Tan propose a fog algorithm by maximizing local contrast[6]. But, such methods are easy to saturate. Fattal proposed that the theoretical propagation of the localized area is irrelevant to the shading part. Whereby the cut-off image is obtained and then the original image is presented[7].This method is liable to cause loss of color information in heavy fog portion, the effect is not ideal. Edwin, land et al proposed Retinex algorithm based on color constancy theory[8].He et al proposed a dark passage theory with priori rule firstly, Calculate a rough projection secondly, recovery the original image by using the guidance filtering algorithm Finally[9].

For the over-enhancement phenomenon of dark channel sky, CHEN et al used the Gaussian function to estimate the transmissivity and eliminated the block effect with the maximum and minimum operation. Then, through the halo operator and the morphology expansion operation to obtain the atmospheric light description area in order to obtain the atmospheric light value, Finally ,according to the atmospheric scattering model to restore a clear image [10],However, this algorithm above need to perform a complex operation, which is not suitable for scenes of the high real-time requirements.

\section{DARK CHANNEL Fog REMOVAL ALgORITHM}

\section{A. Determine the Atmospheric Model}

An atmospheric scattering model is widely used in the field of single image de-fog is shown in equation 1 [12].

$$
I(x)=J(x) t(x)+A(1-t(x))
$$

where, $\mathrm{I}(\mathrm{x})$ is a fog image, $\mathrm{J}(\mathrm{x})$ is the reflection image of the object, $t(x)$ is the transmissivity in the air, and $\mathrm{A}(\mathrm{x})$ is the atmospheric light intensity. The formula shows that the main reason for image degradation is the influence of fog on atmospheric transmissivity $[9,10,11,12]$.

\section{B. Dark Channel Theory}

He et al statistics on large amounts of non fogging images and found that most of the images in non-sky areas with non fogging images have the phenomenon that value in some pixel channel tends to zero.

$$
\mathrm{J}^{\text {dark }}(\mathrm{x})=\min _{\mathrm{c} \in\{\mathrm{r}, \mathrm{g}, \mathrm{b}\}}\left(\min _{\mathrm{y} \in \Omega(\mathrm{x})} \mathrm{J}^{\mathrm{c}}(\mathrm{y})\right) \rightarrow 0
$$

where $J^{\text {dark }}$ is the dark primary, $\Omega(x)$ is the neighborhood of pixel $\mathrm{x}$, and $J^{c}$ is the foggy image. In the image with fog, the value of $J^{\text {dark }}$ becomes high, and the size of the transmittance $\mathrm{t}(\mathrm{x})$ can be reversed [9]. 
Assuming that the atmospheric light value is the constant $\mathrm{A}[9]$, the transmittance is $\mathrm{t}(\mathrm{x})$, the estimated value $\mathrm{t}(\mathrm{x})$ of the transmittance is obtained from some operations by equation 1 .

$$
\tilde{t}(x)=1-\min _{c \in\{r, g, b\}}\left[\min _{y \in \Omega(x)} \frac{I^{c}(y)}{A^{c}}\right]
$$

where $t(x)$ is the estimated value of the transmittance, $c$ is the RGB three-channel space, and $\Omega(x)$ is the filter window space.

He considered that the atmospheric light intensity Ac is obtained from the region with the largest brightness. In this paper, enhanced suppression algorithm uses window scanning to find Ac. This algorithm evaluates the mean of the RGB channel of pixels in the window, Then take the maximum value of all the pixels in the picture as an estimate of the atmospheric light intensity Ac

$$
\mathrm{Ac}=\max \left(\operatorname{mean}_{\Omega}(R), \operatorname{mean}_{\Omega}(G), \operatorname{mean}_{\Omega}(B)\right)
$$

where Ac is the estimate of atmospheric light intensity, $\Omega(\mathrm{x})$ is the window with the largest gray scale. According to the actual requirement, the window size is $15 \times 15$ [9].

The transmittance $\tilde{t}(\mathrm{x})$ and the atmospheric light intensity $\mathrm{A}^{\mathrm{c}}$ can be obtained by the calculation of formula 4-5. According to equation 5 to calculate the original image $\mathrm{J}(\mathrm{x})[9]$.

$$
\mathrm{J}(\mathrm{x})=\frac{I(x)-A^{c}}{\tilde{t}(x)}+A^{c}
$$

where $J(x)$ is a foggy image, $I(x)$ is a fog image, $A^{c}$ is an estimate of atmospheric light intensity, and $\tilde{t}(\mathrm{x})$ is an estimate of transmittance.

\section{PHENOMENON OF OVER-ENHANCEMENT AND ITS IMPROVEMENT}

There is a common problem among algorithms. For example He algorithm [9], Retinex algorithm based on dark-channel theory[2], Single image de-fog algorithm based on dark color and incident light assumption[13], and other dark channel de-fog algorithms . The sky area will emerge lots of stripes or overenhancement phenomenon or color block phenomenon.

The reason for these phenomena is a flaw in the darkchannel algorithm, that is, in the sky area, not all pixels have a channel component that tends to zero. This results in a serious deviation based on the estimation of the transmittance $t$ of the sky region in the dark channel algorithm [9]. As shown in figure 1 , that are phenomena of over-enhancement and color block in the sky.
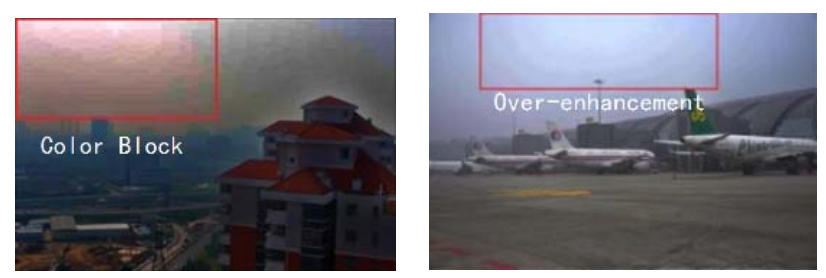

FIGURE I. PROBLEMS IN CLASSICAL ALGORITHMS

\section{A. Sky over Enhancement Suppression Algorithm}

In the foggy picture, RGB value in a pixel of the sky area are basically equal to each other and each value tends to the largest.Some of values of RGB In non-sky areas tend to zero because of influence of object [9]. In order to extract the sky region, a fast limiting algorithm is proposed in this paper. The result of fast limiting algorithm is used to correct the transmittance of the sky region inthe dark channel algorithm, inhibit over-enhanced phenomenon in the sky area.

1) Histogram equalization: The essence of the limiting algorithm is to extract the region where the RGB values are basically equal to each other and the values of each channel tend to the largest. The gray scale information of the region is retained simultaneously. Because of the picture affected by the fog, histogram of each channel are too concentrated to extract the gray-scale information. So, image equalization is necessary, which mainly includes RGB Direct Method and HSV Transformation Method. The HSV transformation can avoid color distortion [14].Contrast found that in the fog picture, the difference value between the absolute value of the difference value between the two is not big. As showed in Figure 2, the two methods are compared. Where the different value of the absolute value is:

$$
\text { err }=\left|H_{R G B}-H_{H S V}\right|
$$

where err represents the absolute value of the difference between the RGB Direct Method and the HSV Method. HRGB represents an image obtained based on RGB equalization. HHSV represents an image obtained based on HSV equalization.

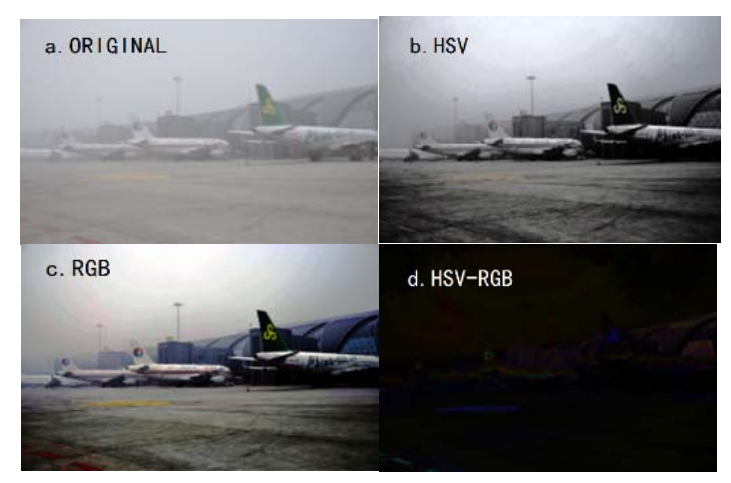

FIGURE II. COMPARING ALGORITHM

Statistics of 493 pictures with fog in sky areas by using equation 7 to calculate the average value of the absolute difference of two algorithms. The distribution of its absolute value is shown in Table 1.

$$
\operatorname{Err}=\{\operatorname{mean}[\operatorname{err}(\mathrm{R})], \operatorname{mean}[\operatorname{err}(\mathrm{G})], \operatorname{mean}[\operatorname{err}(\mathrm{B})]\}
$$

where, Err is a three-dimensional vector; $\operatorname{Err}(\mathrm{R}), \operatorname{Err}(\mathrm{G}), \operatorname{Err}(\mathrm{B})$ respectively represent the RGB three channel sub-matrix in the deviation matrix; mean is the mean operation.

According to the Table 1, the absolute difference value in $90 \%$ images with fog between these two algorithms is less than 
8. Considering the long operation time of RGB-HSV transform and inverse transform, the image equalization algorithm based on RGB 3-channel achieve good results.

TABLE I. MEAN DISTRIBUTION OF THE ABSOLUTE DIFFERENCE

\begin{tabular}{|l|l|l|l|l|l|}
\hline difference & $0-2$ & $2-4$ & $4-6$ & $6-8$ & $>8$ \\
\hline R channel & 230 & 133 & 60 & 28 & 42 \\
\hline G channel & 216 & 104 & 83 & 46 & 44 \\
\hline B channel & 248 & 108 & 39 & 37 & 61 \\
\hline
\end{tabular}

2) Validity analysis of limiter algorithm: If the gray region information of the sky region is supersaturated, values of three channels of the single pixel need to be mapped to a value e.

$$
e=\lim 2\left\{\min \left(E_{i}, E_{j}, E_{k}\right)-\mu \times \operatorname{Lim} 1\left[E_{i}+E_{j}+E_{k}\right]\right\}
$$

where e is the output value; $\mu$ is the compression factor; $E_{i}, E_{j}$ and $E_{k}$ are three different channel values in the single pixel; Lim1, Lim2 are limiter, where output of limiter is that less than $L_{i m}$ min equal to $L_{i m_{m i n}}$, greater than $\operatorname{Lim}_{\max }$ equal to $\operatorname{Lim}_{\max }$. Considering the depth of pictures is 8 , let $\operatorname{Lim} 2_{\min }=$ $0, \operatorname{Lim} 2_{\max }=255$.

In the formula $8, \operatorname{Lim} 1\left[E_{i}+E_{j}+E_{k}\right]=\left(E_{i}+E_{j}+E_{k}\right)>$ $3 \times \min \left(E_{i}, E_{j}, E_{k}\right)$. Compression factor $\mu>1 / 3$ to make e equals zero when $\left(E_{i}+E_{j}+E_{k}\right)$ does not exceed the upper limit of the limiter.

$\operatorname{Lim} 1\left[E_{i}+E_{j}+E_{k}\right]$ is a fixed value when $\left(E_{i}+E_{j}+\right.$ $\left.E_{k}\right)$ exceeds the upper limit of the limiter. And the value of $\min \left(E_{i}, E_{j}, E_{k}\right)$ where the RGB three channels not tend to zero is greater than the value of $\min \left(E_{i}, E_{j}, E_{k}\right)$ where a certain channel of RGB tends to zero; When the compression factor $\mu$ is increased, the value of $\min \left(E_{i}, E_{j}, E_{k}\right)-\mu \times \operatorname{Lim} 1\left[E_{i}+\right.$ $\left.E_{j}+E_{k}\right]$ does not tend to zero. If the one or two channels of the RGB channel become zero, the the value of $\min \left(E_{i}, E_{j}, E_{k}\right)-$ $\mu \times \operatorname{Lim} 1\left[E_{i}+E_{j}+E_{k}\right]$ decreases to zero

In summary, the increase of the compression factor $\mu$ can filter one or two channels in the pixel tends to zero. A reasonable choice of compression factor $\mu$, The gray information where RGB channels not tend to zero can be got.

3) Experimental Verification: A fog picture Randomly is selected in this experiment, The range of Lim1 is 0255. $E_{i}, E_{j}, E_{k}$ are respectively blue, green and red channel. Results from Figure 3(a) to (d) are based on different compression factor $\mu$.

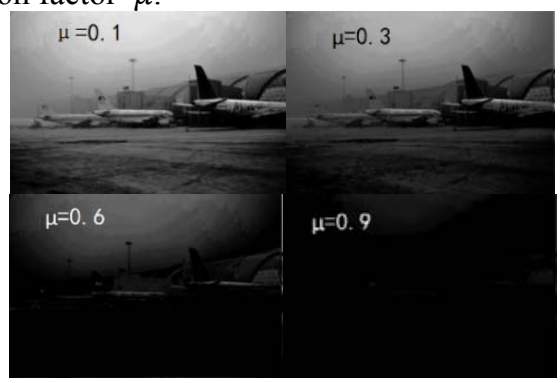

FIGURE III. RESULT FROM DIFFERENT COMPRESSION FACTOR M
The limiter Lim1 is related to the compression factor $\mu$. Different limiter Lim1 with an appropriate $\mu$ be selected to achieve a similar effect. As showed in Figure 4, results from the combination of different Lim1 and different $\mu$.

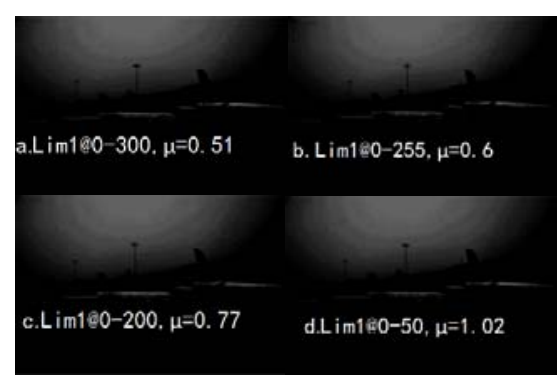

FIGURE IV. RESULTS FROM DIFFERENT LIM1 AND DIFFERENT M

It can be seen from Figure. 4 that the effects of the four graphs are similar. Table 2 will be got by using formula 9 .

$$
\mathrm{G}=\frac{\sum_{x \in \Omega}\left(T_{i}(x)-T_{j}(x)\right)}{w \times h} \times \frac{1}{255}
$$

where $\mathrm{G}$ is the average gray scale; $\Omega$ is the picture space; $T_{i}(x)$ are gray value in the picture space in Figure. $4 . \mathrm{a} ; T_{j}(x)$ are gray value in the picture space in Figure.4.b- Figure.4.d. $w$ is the image width, $h$ is the picture height.

It can be seen from the Table 2that the differences between the four images in Figure. 4 are are below 10\%. Statistics of 493 pictures with fog in sky areas by using equation 9 shows that More than $95 \%$ of the pictures are below $10 \%$.

TABLE II. AVERAGE GREY VALUE

\begin{tabular}{|c|c|c|c|}
\hline Image & Figure.4.a-4.b & Figure.4.a-4.c & Figure.4.a-4.d \\
\hline Ratio & $1.8 \%$ & $4.3 \%$ & $5.9 \%$ \\
\hline
\end{tabular}

In order to work fast in computers, our algorithm uses the overflow feature of the 8-bit unsigned integer operation instead of the judgment statement. This algorithm is computationally less and the validity of the results is guaranteed. So, selected range of two limiter is $0-255$.

\section{B. Best Compression Factor}

In this paper, the new visible edges ratio[15] is used as the evaluation index. When $\mu$ is increased from 0 , there is no obvious change of visible edges in the sky area, The single continuous visible edges in ground region and boundary region are first split into multiple small visible edges then be removed. It is obvious that the total number of edges increases at first and then decreases. When the number of visible edges reaches the inflection point, the visible edges in ground area are removed, while the boundary region retains some visible edges. It is found that when the boundary region retains some visible edges, it can suppress the over enhancement of the boundary region and retain more details. Therefore, the $\mu$ is the best when the inflection point appears on the number of visible edges.

Best compression factor of fog pictures in 493 images with fog in sky area are count in Table 3, The statistical results show that the amplitude limit of the two limiters is $0-255$, the best range of compression factor $\mu$ is $0.5-0.6$. 
TABLE III. OPTIMAL COMPRESSION FACTOR DISTRIBUTION

\begin{tabular}{|c|c|c|c|c|c|c|}
\hline range & $0.2-0.3$ & $0.3-0.4$ & $0.4-0.5$ & $0.5-0.6$ & $0.6-0.7$ & $0.7-0.8$ \\
\hline count & 2 & 7 & 60 & 277 & 90 & 57 \\
\hline
\end{tabular}

\section{Transmittance Correction}

According to the dark channel theory, the transmittance of the pixels where all RGB values not near zero is too large[9]. The estimated transmittance value $\tilde{t}(\mathrm{x})$ is corrected using the Grey information (e) of the failure region in dark channel theory.

$$
\tilde{t}(x)=\tilde{t}(x)-k \times e
$$

where $\tilde{\tilde{t}}(\mathrm{x})$ is the correction value of the transmittance estimate $\tilde{t}(\mathrm{x}) ; \tilde{t}(\mathrm{x})$ is the transmittance estimate obtained by the dark channel algorithm; e is the correction matrix obtained by the equation $8, \mathrm{k}$ is the correction factor.

The magnitude of the $\mathrm{k}$ value affects the transmittance of the dark channel failure region. Experiments show that the selection of inappropriate $\mathrm{k}$ value will affect the uniformity of gray area in the sky area.

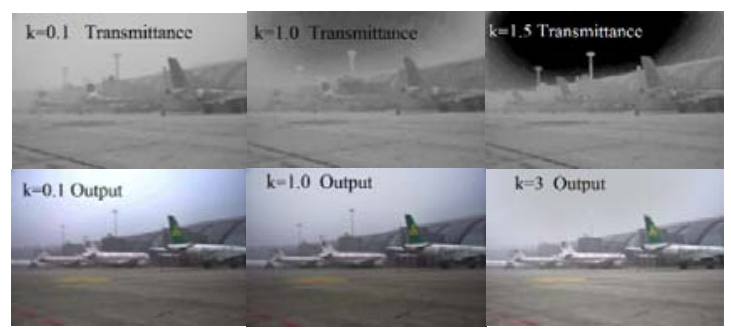

FIGURE V. TRANSMITTANCE OF THE SKY REGION

As shown from Figure 5, when $\mathrm{k}$ value increases, the transmittance of the sky region decreases and the uniformity becomes better. However, if the $\mathrm{K}$ value increased too large, the details of the sky region will be lost. Our experimental results show that when $\mathrm{k}$ is $1.1-1.3$, the sky uniformity is the best, the supersaturated pixel[13] is low. Taking into account the time complexity, regardless of the adaptive $\mathrm{k}$ value, our algorithm makes $\mathrm{k}=1.2$.

\section{EXPERIMENTAL RESULTS AND ANALYSIS}

The algorithm contrast effect experiment is carried out by using figures 6.a and 7.a. Where parameters is selected as below: The minimum atmospheric light value is 240 , the bootstrap filter window and the minimum filter window are $15 \times 15$, the error limit is 10-6. The experimental results are shown in Figures 6 to 7.

As is shown from the comparison of the two algorithms in Figure 6. Compared with the He algorithm , our algorithm suppress the over-enhancement in the sky area effectively.At the same time the gray scale of the sky region is more uniform and retain more details.

Figure 7 compares two algorithms, and shows that our algorithm suppress the phenomenon of color blocks effectively.

In this paper, the method of visible side is used to evaluate the He algorithm and the enhanced suppression algorithm. This method calculates the percentage of new visible margin e, normalized gradient ratio $\overline{\mathrm{r}}$, the percentage of oversaturated black or white pixels $\sigma[13]$.

$$
\left\{\begin{array}{l}
e=\frac{n_{1}-n_{0}}{n_{0}} \\
r=\frac{\Delta I_{1}}{\Delta I_{0}} \bar{r}=\exp \left[\frac{1}{n_{1}} \sum_{x_{1}} \log r_{i}\right] \\
\sigma=\frac{n^{\prime}}{w \times h}
\end{array}\right.
$$

where $\mathrm{n}_{0}$ is the number of visible edges, $\mathrm{n}_{1}$ is the number of visible edges of the graph after processing, $r$ is the visible correlation coefficient, $\Delta \mathrm{I}_{1}, \Delta \mathrm{I}_{0}$ are gradient of pixels on visible edges of the fog map and the original image respectively. $\overline{\mathrm{r}}$ is the balanced gradient ratio; $\mathrm{x}_{1}$ is the pixel point on the edge. $\sigma$ is the oversaturated pixel ratio, $\mathrm{n}^{\prime}$ is the number of supersaturated pixels.

The defogging effect is better when the values of $E$ and $\bar{r}$ are bigger at the same time $\sigma$ is smaller [15]. Table 4 compares the He algorithm with our algorithm.

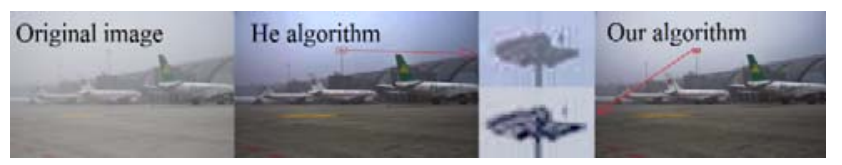

FIGURE VI. ENHANCED SUPPRESSION ALGORITHM AND LOCAL DETAILS

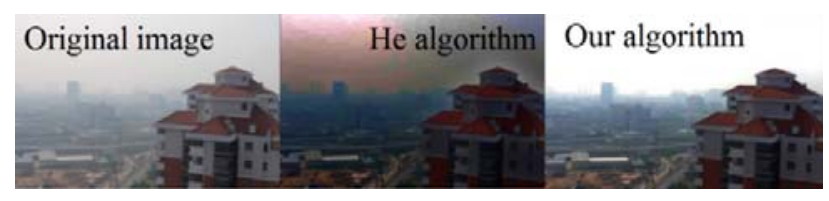

FIGURE VII. COLOR BLOCK SUPPRESSION

TABLE IV. COMPARISON OF HE ALGORITHM AND OUR ALGORITHM

\begin{tabular}{|c|c|c|c|c|}
\hline \multirow{2}{*}{ Img } & \multirow{2}{*}{ Algorithm } & \multicolumn{3}{|c|}{ index } \\
\cline { 3 - 5 } & & $\mathbf{e}$ & $\overline{\mathbf{r}}$ & $\boldsymbol{\sigma}$ \\
\hline \multirow{2}{*}{ Figure 6 } & $\mathrm{He}$ & 2.1336 & 1.6618 & 0.0651 \\
\cline { 2 - 5 } & Our & 2.0994 & 1.6178 & 0.0001 \\
\hline \multirow{2}{*}{ Figure 7 } & He & 0.4118 & 0.8314 & 0.2336 \\
\cline { 2 - 5 } & Our & 0.3547 & 0.7811 & 0.0018 \\
\hline
\end{tabular}

From Table 4 shows that our algorithm has a great improvement over the He algorithm in the supersaturated pixel ratio $\sigma$, but the e and the $\overline{\mathrm{r}}$ of our algorithm is slightly reduced. Statistics of 493 pictures with fog in sky areas by using equation 11 showed a reduction of $99 \%$ in ratio $\sigma$ over $80 \%$ of these pictures.

\section{CONCLUSION}

Aiming at restrain the phenomenon that pixels in sky area is easy to be enhanced in the He de-fog algorithm, in this paper a correction algorithm is proposed. Our algorithm is simple and dispense with edge detection, corrosion, expansion or other complex operations. The transmission rate was corrected by using a straight - square - balanced gray-scale correction matrix. Experiments show that our algorithm solve the problem more 
effectively by comparing with He algorithm. The sky area is more uniform and no color blocks appear.

\section{REFERENCES}

[1] Sheng H L, Isa N A M, Chen H O, et al. A new histogram equalization method for digital image enhancement and brightness preservation[J]. Signal Image \& Video Processing, 2015, 9(3):675-689.

[2] Yeonan-Kim J, Bertalmío M. Analysis of retinal and cortical components of Retinex algorithms[J]. Journal of Electronic Imaging, 2017, 26(3).

[3] Ancuti C O, Ancuti C. Single image dehazing by multi-scale fusion.[J]. IEEE Transactions on Image Processing A Publication of the IEEE Signal Processing Society, 2013, 22(8):3271-3282.

[4] Wang J B, He N, Zhang L L, et al. Single image dehazing with a physical model and dark channel prior[J]. Neurocomputing, 2015, 149(PB):718728.

[5] Ju M, Zhang D, Wang X. Single image dehazing via an improved atmospheric scattering model[J]. Visual Computer, 2016:1-13.

[6] Tan R T. Visibility in bad weather from a single image[C]// Computer Vision and Pattern Recognition, 2008. CVPR 2008. IEEE Conference on. IEEE, 2008:1-8.

[7] Fattal R. Single image dehazing[C]// ACM, 2008:1-9.

[8] Land E H. The Retinex Theory of Color Vision[J]. Scientific American, 1977, 237(6):108.

[9] He K, Sun J, Tang X. Guided image filtering[J]. Pattern Analysis \& Machine Intelligence IEEE Transactions on, 2013, 35(6):1397.

[10] G Chen, Y Yang, B Zhang. Fog removal algorithm based on improved dark prior prior to transmittance and atmospheric light(in chinese)[J].Journal Of Computer Applications, 2017, 37(5):1481-1484.

[11] Deng Li. Adaptive global dark prior prior to fog removal for bright regions(in chinese)[J]. Optics and Precision Engineering, 2016, 24(4):892-901.

[12] Makkar D, Malhotra M. Single Image Haze Removal Using Dark Channel Prior[J]. International Journal of Advanced Trends in Computer Science \& Engineering, 2016.

[13] MinJie Yu, Haofeng Zhang.Single image de fog based on dark color and incident light assumption(in chinese)[J] Journal of Image and Graphics , 2014, 19(12):1812-1819.

[14] Bora D J, Gupta A K. AERASCIS: An efficient and robust approach for satellite color image segmentation[C]// International Conference on Electrical Power and Energy Systems. IEEE, 2017:549-556.

[15] Hautière N, Tarel J P, Aubert D, et al. Blind Contrast Enhancement Assessment by Gradient Ratioing at Visible Edges[J]. Image Analysis \& Stereology, 2008, 27(2):87-95. 